\title{
Análisis de la coordinación motriz global en escolares según género, edad y nivel de actividad física Analysis of global motor coordination in schoolchildren according to gender, age and level of physical activity \\ Andrés Rosa Guillamón, Eliseo García Canto, Hector Martínez García \\ Universidad de Murcia (España)
}

\begin{abstract}
Resumen. El objetivo fue analizar la coordinación motriz según género, edad y nivel de actividad física. Estudio descriptivo-transversal realizado con 101 escolares de 6-8 años. Se midió la coordinación motriz con el Test 3JS, y la actividad física con el Test corto de actividad física Krece Plus. Los análisis de la varianza simple arrojaron los siguientes resultados: 1) los varones tuvieron un mejor desempeño motor que las mujeres en lanzamiento de precisión, golpeo de precisión, bote y conducción ( $p$ entre $<.05 \mathrm{y}<.001)$, mostrando una mejor coordinación motriz global $(p=.002)$ y eficacia coordinativa $(p<.001) ; 2)$ los escolares de 8 años tuvieron un mejor desempeño motor que sus semejantes de 6-7 años en giro, bote y conducción $(p<.001)$, mostrando una mejor coordinación motriz global y eficacia coordinativa ( $p<.001$ para ambos); 3 ) aquellos categorizados como más activos tuvieron un mejor desempeño motor que sus semejantes menos activos en bote y conducción $(p=.001$ para ambos), mostrando también una mejor coordinación motriz global y eficacia coordinativa ( $p=.001$ para ambos). Los análisis de regresión lineal mostraron la capacidad predictiva del género, la edad y la actividad física sobre la coordinación motriz. Los resultados sugieren que tener un mayor nivel de actividad física, ser varón y tener una mayor edad son tres elementos diferenciadores de la coordinación motriz en escolares españoles de seis a ocho años.
\end{abstract}

Palabras clave: actividad física, educación física, coordinación motriz, eficacia coordinativa, escolares.

\begin{abstract}
The objective was to analyze the motor coordination according to gender, age and level of physical activity. Descriptive crosssectional study carried out with 101 schoolchildren aged 6-8 years old. Motor coordination was measured with the 3JS Test, and physical activity with the Krece Plus Short Physical Activity Test. The analysis of the simple variance generated the following results: 1) boys had better motor performance than girls in precision throwing, precision hitting, ball jump, and ball movement ( $\mathrm{p}$ between $<.05$ and $<.001)$, showing better global motor coordination $(\mathrm{p}=.002)$ and coordination effectiveness $(\mathrm{p}<.001)$; 2$)$ 8-year-old schoolchildren had better motor performance than 6-7 year-old schoolchildren in ball spin, ball jump, and ball movement $(\mathrm{p}<.001)$, showing better overall motor coordination and coordinating effectiveness ( $<.001$ for both); 3 ) those categorized as more active had better motor performance than their less active peers in ball jump and ball movement $(\mathrm{p}=.001$ for both), also showing better overall motor coordination and coordination effectiveness $(\mathrm{p}=.001$ for both). Linear regression analysis showed the predictive capacity of gender, age and physical activity on motor coordination. The results suggest that having a higher level of physical activity, being male and being older are three differentiating elements for motor coordination in Spanish schoolchildren aged six to eight years old.
\end{abstract}

Keywords: physical activity, physical education, motor coordination, coordination effectiveness, school children.

\section{Introducción}

La coordinación motriz hace referencia a las capacidades perceptivo-cinéticas que permiten integrar los factores motores sensitivos y sensoriales que facilitan la organización y la regulación de las acciones motrices necesarias para desarrollar una tarea motriz con precisión, economía, armonía y eficacia, en un proceso de interacción entre la persona y el entorno (Lladó, 2017).

La coordinación motriz es uno de los componentes más relevantes de la competencia motriz y que mejor se relaciona con la condición física relacionada con la salud (Ruiz-Pérez, Navia, Ruiz, Ramón, \& Palomo, 2015). La coordinación motriz se compone a su vez de otras dimensiones o expresiones que engloban el acto motor, mediante las cuales se persigue un objetivo motor determinado (Cenizo, Ravelo, Morilla, \& Fernández, 2017),

La coordinación motriz ha sido analizada en la literatura científica desde distintas perspectivas (Torralba, Vieira, Lleixá, \& Gorla, 2016). Meinel \& Schnabel (2013) se han centrado en el estudio de la coordinación motriz desde las perspectivas pedagógica, fisiológica y biomecánica, coincidiendo con teorías más recientes donde se relacionan diferentes capacida-

Fecha recepción: 15-08-19. Fecha de aceptación: 30-11-19

Eliseo Garcia Canto

eliseo.garcia@um.es des motrices con elementos perceptivo-coordinativos (Gorla, Araújo, \& Rodrigues, 2010). Otros trabajos han estudiado la coordinación motriz en relación con variables tales como el rendimiento académico (Ruiz-Pérez, Navia, Ruiz, Ramón, \& Palomo, 2016), el morfotipo (Freitas et al., 2015), el estatus social y la composición corporal (Bucco \& Zubiaur, 2015; Hardman, Júnior, de Souza, Oliveira, \& Barros, 2017), el desarrollo motor (Ruiz-Pérez, et al., 2015), la actividad física (De Meester, et al., 2016) o las capacidades condicionales (Yanci \& Los Arcos, 2015),

Los resultados de algunos estudios indican que un desarrollo adecuado de la coordinación motriz resulta primordial en la maduración física, motriz y cognitiva en edades prepuberales (Coetzee, 2016; Walhain, van Gorp, Lamur, Veeger, \& Ledebt, 2016). Asimismo, se ha observado que variables como el equilibrio, la fuerza y la agilidad son esenciales para la eficacia coordinativa en la realización de actividad física (Pienaar, et al., 2012; Rosa \& García-Cantó, 2017; Ru•barská, 2016).

En este sentido, se ha descrito que niveles reducidos de actividad física se encuentran relacionados con un bajo nivel de coordinación motriz (Jarani, et al., 2016; Ruiz-Pérez, et al., 2015). Se ha constatado que tienen mayor riesgo de hipoactividad aquellos escolares que manifiestan trastorno del desarrollo de la coordinación (Cairney, et al., 2005b), afectando entre el $5 \%$ y el $9 \%$ de sujetos en edad prepuberal, siendo reconocido como uno de los principales trastornos 
relacionados con el desarrollo motor (Cairney, Hay, Faught, Mandigo, \& Flouris, 2005a). Además, estos escolares que se perciben poco eficaces motrizmente o que tienen una autocompetencia deportiva baja presentan niveles reducidos de actividad física extraescolar (De Meester, et al., 2016). Esta inactividad física puede incrementar el sobrepeso y favorecer un nivel bajo de condición física relacionada con la salud (Pérez-Soto, et al., 2018), lo cual puede aumentar el riesgo de padecer enfermedades cardiovasculares en edades futuras (Cairney, 2015; Secchi, García, España-Romero, \& Castro Piñero, 2014).

Por estas y otras causas, la coordinación motriz se ha convertido en uno de los contenidos más importantes a trabajar en la escolarización obligatoria, encontrándose reflejada en los currículos diseñados por las diferentes administraciones educativas (López, Lara, Espejo, \& Cachón, 2016; Torralba, et al., 2016). El tratamiento de la coordinación motriz en el ámbito educativo es básico no solo para evitar un posible deterioro físico, sino también para favorecer un desarrollo integral del escolar a nivel motor, cognitivo y socioafectivo (Bravo, Rodríguez-Negro, \& Irigoyen, 2017).

El desarrollo de la coordinación motriz se encuentra integrado en el trabajo de la competencia motriz global, siendo especialmente importante abordar esta cuestión desde el área de educación física, donde los escolares que muestran niveles elevados de disfrute en las clases muestran un mejor desarrollo coordinativo (Hay, 1996) y una mayor percepción de autoeficacia deportiva (Ruiz-Pérez, et al., 2015); lo que puede influir a su vez en la adopción de la actividad física como un hábito de estilo de vida que favorece la mejora de la condición física relacionada con la salud (De Miguel-Etayo, et al., 2014; Rosa, et al., 2017; Torres-Luque, Carpio, Lara, \& Zagalaz, 2014). Este hecho es relevante ya que la condición física ha sido relacionada en escolares con niveles superiores de rendimiento académico (Rosa, García, \& Carrillo, 2019), bienestar emocional (Rosa, García, \& Pérez, 2018) y autopercepción de salud (Rosa, García-Cantó, \& Carrillo, 2018).

En el tratamiento adecuado de la coordinación motriz desde la edad escolar puede ser importante tener en cuenta variables como el género (Bravo, et al., 2017; Marta, Marinho, Barbosa, Izquierdo, \& Marques, 2012; Rodríguez-Negro \& Yanci, 2019; Yanci, Los Arcos, Reina, Gil, \& Grande, 2014) y la edad (Fernández-Losa, Cecchini, \& Pallasá, 2013; CarrilloLópez, Rosa-Guillamón, \& García-Cantó, 2018).

Algunos estudios revelan que los varones tienen mayor predisposición a presentar trastornos coordinativos que las mujeres (Kokštejn, Psotta, \& Musálek, 2015), aunque estas diferencias suelen ser mínimas en las edades tempranas (Lingam, Hunt, Golding, Jongmans, \& Emond, 2009); haciéndose más evidentes conforme aumenta la edad, presentando los varones mejores capacidades coordinativas que las mujeres (Barnett, Van Beurden, Morgan, Brooks, \& Beard, 2010; Butterfield, Angell, \& Mason, 2012). Sin embargo, otros estudios no han encontrado diferencias en la coordinación motriz (Coetzee \& Du Plessis, 2013), o bien dichas diferencias están repartidas entre ambos sexos en las diferentes pruebas analizadas (Coetzee, 2016).

En otros estudios, se ha concluido que capacidades relacionadas con la coordinación motriz tales como la velocidad, la agilidad y la fuerza suelen ser similares hasta los 7 años de edad, siendo entre los 8 y los 12 años cuando los varones mejoran estas capacidades en comparación a las mujeres (Gallahue \& Ozmun, 2006); aunque existen estudios que contradicen estos resultados (Lazzer, Pozzo, Rejc, Antonutto, \& Francescato, 2009; Tekok-Kiliç, Elmastaç-Dikeç, \& Can, 2010) o reportan en los varones mejores valores en determinadas capacidades coordinativas como, por ejemplo, la coordinación óculo manual (Carrillo-López, Rosa-Guillamón, \& GarcíaCantó, 2018; Singh, Dhanda, \& Shanwal, 2010).

Por la complejidad del proceso madurativo de la coordinación motriz, se ha establecido la educación primaria como la etapa primordial para el desarrollo progresivo de este conjunto de capacidades coordinativas, debido a que en este periodo se produce un gran desarrollo físico, así como la madurez y el incremento de la eficacia de las habilidades motrices básicas, estrechamente relacionadas con dichas capacidades (Cenizo, Ravelo, Morilla, Ramírez, \& FernándezTruan, 2016; Jones \& Lorenzo, 2013). Fruto de este interés se han incrementado las investigaciones desde los ámbitos científico y educativo con el objetivo de evaluar las capacidades coordinativas en edades prepuberales (Comuk-Balci, et al., 2016; Ericsson, 2011; Ericsson \& Karlsson, 2014; Hardman, Júnior, de Souza, Oliveira, \& Barros, 2017; Jarani, et al., 2016; Lopes, Maia, Silva, Seabra, \& Morais, 2003; Tekok-Kiliç, et al., 2010; Torralba, et al., 2016; Yanci \& los Arcos, 2015; Yanci, Los Arcos, Reina, Gil, \& Grande, 2014; Walhain, van Gorp, Lamur, Veeger, \& Ledebt, 2016).

Para realizar esta evaluación existen diferentes instrumentos, desde los más tradicionales como el Perfil Psicomotor (Picq \& Vayer, 1977) o el KTK (Kiphard \& Schilling, 1974) hasta los más novedosos como el revisado MABC-2 (Henderson, Sudgen, \& Barnett, 2007) o el Test Motor GRAMI-2 (Ruiz-Pérez, Rioja-Collado, Graupera-Sanz, Palomo-Nieto, \& García-Coll, 2015). Para este estudio se ha elegido el Test 3JS de Cenizo, et al. (2016) ya que permite analizar no solo la coordinación motriz global (Cenizo, et al., 2015) sino también dos de sus expresiones, la coordinación locomotriz y la coordinación control de objetos con las manos y con los pies (Cenizo, et al., 2017), facilitando de esta manera el análisis completo de los factores que pueden influir en su desarrollo y en la detección de escolares con trastorno del desarrollo de la coordinación en las primeras fases evolutivas.

Sobre la base de lo anterior, el objetivo de este estudio fue analizar la coordinación motriz según el género, la edad y el nivel de actividad física en una muestra de escolares de educación primaria.

\section{Material y método}

\section{Diseño y participantes}

Un total de 101 escolares (55 varones y 46 mujeres) de la Región de Murcia (España) con una edad comprendida entre los seis y los ocho años de edad $(M \pm D T: 6.93 \pm 0.73$ años) participaron en este estudio descriptivo transversal. Los participantes residían en un entorno urbano y pertenecían a un colegio público. Los participantes fueron elegidos de manera no aleatoria y por conveniencia. Se solicitó el consentimiento informado familiar. Se planteó como criterio 
de exclusión tener una patología neurológica o músculoesquelética. La investigación respetó los postulados de la Declaración de Helsinki de 2013, y recibió la aprobación de la Comisión de Ética de la Universidad de Murcia (cuatro de diciembre de dos mil dieciocho).

\section{Instrumentos}

La actividad física fue evaluada con el Test corto de actividad física Krece Plus, perteneciente al estudio en Kid, desarrollado entre los años 1998 y 2000 (Román, Serra, Ribas, Pérez, \& Aranceta, 2003). El test permite registrar la media diaria de horas que los escolares ven la televisión o practican con videojuegos (ítem 1), y las horas semanales de actividad física extraescolar (ítem 2). Las puntuaciones de cada ítem oscilan entre cero y cinco (en el ítem 1: cero horas $=5$, una hora $=4$, dos horas $=3$, tres horas $=2$, cuatro o más horas $=1$; en el ítem 2: cero horas $=0$, una hora $=1, \operatorname{dos}$ horas $=2$, tres horas $=3$, cuatro o más horas $=4$ ). A partir de estos valores se creó la variable actividad física como la media de ambos ítems. Se definió un grupo menos activo cuando el nivel de actividad física fue $\mathrm{X}<\mathrm{P}_{50}$, y más activo cuando el nivel de actividad física fue $\mathrm{X}$ e» $\mathrm{P}_{50}$ (el $\mathrm{P}_{50}$ fue de 3.1 puntos). El alpha de Cronbach del instrumento fue de .70.

Para medir la coordinación motriz se empleó el Test 3JS (Cenizo, et al., 2016). Este instrumento permite evaluar la coordinación motriz global desde una perspectiva criterial-cualitativa. La evaluación del instrumento se realiza mediante la observación visual de la ejecución práctica de siete pruebas que se realizan de forma continua y sin descanso. Cada prueba es calificada mediante una escala descriptiva con cuatro niveles de desempeño, siendo uno el desarrollo más inmaduro y cuatro el desarrollo más maduro (tabla 1). Las pruebas permiten configurar factores que evalúan no solo la coordinación motriz en su globalidad sino también expresiones de esta capacidad: 1) coordinación locomotriz (CL): salto a pies juntos, giro sobre el eje longitudinal y carrera de eslalon; 2) coordinación control de objetos (CCO): lanzamiento de balón, golpeo de pelota, bote de balón y conducción de balón; 3) coordinación control de objetos con el pie (CCOP): golpeo de balón y conducción de balón; 4) coordinación control de objetos con la mano (CCOM): lanzamiento de balón y bote de balón; 5) CMG (CMG): media de los valores obtenidos en cada una de las variables de la coordinación motriz (Cenizo, et al., 2017). La fiabilidad del test es elevada (á $=.827$ ) y la estabilidad temporal test-retest, con un intervalo de siete días, también $(\mathrm{CCI}=.99)($ Cenizo, et al., 2016).

Se registró también el tiempo de ejecución en el test motor y se calculó la variable eficacia coordinativa, que permite evaluar la eficacia del escolar en tareas que requieren la utilización conjunta de las capacidades coordinativas, con un tiempo empleado según un objetivo determinado.

$$
E A=\frac{C M G}{T \cdot{ }^{\circ}}
$$

Donde (EA) es la eficacia coordinativa, $(\mathrm{CMG})$ es la coordinación motriz global y $\left(\mathrm{T}^{\circ}\right)$ es el tiempo empleado en el test motor. Con la incorporación del factor tiempo se complementa la capacidad del sujeto de organizar y regular las distintas expresiones de la coordinación motriz (locomotriz y control de objetos), con la precisión y producción de los sujetos según el tiempo empleado (eficacia coordinativa).

Tabla 1.

\begin{tabular}{|c|c|}
\hline Prueba & Descripción \\
\hline Salto vertical. & Saltar tres picas con los dos pies juntos. \\
\hline Giro longitudinal. & Saltar y hacer un giro sobre el eje longitudinal entre $1^{\circ}$ y $360^{\circ}$. \\
\hline Lanzamiento de precisión. & $\begin{array}{l}\text { Lanzar dos pelotas al poste de una portería desde una distancia y } \\
\text { sin salirse del cuadro. }\end{array}$ \\
\hline Golpeo de precisión. & $\begin{array}{l}\text { Golpear dos balones al poste de una portería desde una distancia } \\
\text { y sin salirse del cuadro. }\end{array}$ \\
\hline Eslalon. & Desplazarse corriendo en zig-zag entre picas. \\
\hline Bote de balón. & Botar un balón de baloncesto ida y vuelta en zig-zag entre picas. \\
\hline Conducción de pelota. & Conducir un balón ida y vuelta en sentido circular. \\
\hline
\end{tabular}

Fuente: elaboración propia

\section{Procedimiento}

Se solicitó la colaboración en el estudio tanto del equipo directivo como de la asociación de padres. El trabajo de campo se realizó en diciembre de 2018, en horario lectivo, administrando las pruebas en los grupos escolares establecidos por el centro (grupos naturales de clase). El equipo de investigadores estuvo formado por dos maestros especialistas en educación física entrenados para la aplicación del test motor. Se recomendó a las familias que los escolares no realizasen ejercicio físico intenso la tarde anterior. Los escolares realizaron el test motor con ropa deportiva ligera. Se realizó una demostración de cada tarea y un calentamiento con manejo de móviles. El test de actividad física fue autoadministrado en presencia de un investigador para resolver posibles dudas. Todos los test del estudio fueron administrados en una única jornada lectiva.

\section{Análisis estadístico}

Se realizó un análisis descriptivo mediante el cálculo de la media y la desviación típica. El análisis de normalidad se realizó mediante la prueba de Kolmogorov-Smirnov. Las diferencias entre grupos se analizaron mediante un análisis de la varianza simple (ANOVA) para las variables continuas y el test Chi-cuadrado para las variables categóricas. La prueba de Levene fue utilizada para comprobar la homogeneidad de las varianzas. Se realizó un análisis de regresión lineal para estudiar la relación entre actividad física, $\mathrm{CMG}$ y eficacia coordinativa. Los análisis se realizaron de forma separada para las variables de CMG y eficacia coordinativa, y se ajustaron (covariables) por género (varones $=0$, mujeres $=1$ ) y edad (años). El nivel de significación se estableció en el 5\% ( $p \mathrm{~d} »$.05). Se empleó el programa SPSS v.23.0.

\section{Resultados}

La tabla 2 muestra el análisis descriptivo y las diferencias en las variables de estudio según sexo (valor de $p$ e intervalo de confianza). La prueba ANOVA detectó diferencias significativas en las variables lanzamiento de precisión $(p=.001)$, golpeo de precisión $(p=.019)$, bote $(p<.001)$, conducción $(p<.001), C C O(p<.001), C C O P(p<.001), C C O M(p<.001)$, CMG $(p=.002)$, tiempo de ejecución $(p<.001)$ y eficacia coordinativa $(p<.001)$. La prueba Chi-cuadrado no arrojó diferencias en el nivel de actividad física según género.

Al dividir la muestra en tres grupos según la edad, la prueba ANOVA detectó diferencias significativas en las variables giro longitudinal $(p<.001)$, bote $(p<.001)$, conducción $(p<.001), C L(p=.025)$, CCO $(p<.001)$, CCOP $(p$ $<.001), \operatorname{CCOM}(p=.002), C M G(p<.001)$, tiempo de ejecu- 
Tabla 2 .

Diferencias en las variables de estudio según género.

\begin{tabular}{|c|c|c|c|}
\hline & $\begin{array}{l}\text { Varones } \\
(\mathrm{n}=55)\end{array}$ & $\begin{array}{l}\text { Mujeres } \\
(\mathrm{n}=46)\end{array}$ & \\
\hline & $\mathrm{M} \pm \mathrm{DT}$ & $\mathrm{M} \pm \mathrm{DT}$ & $p$ valor \\
\hline \multicolumn{4}{|l|}{ Pruebas del test motor } \\
\hline Salto vertical & $3.2 \pm 1.1$ & $3.4 \pm 0.9$ & .457 \\
\hline (IC 95\%) & $(2.9-3.5)$ & $(3.0-3.6)$ & \\
\hline Giro longitudinal & $2.4 \pm 0.8$ & $2.3 \pm 0.9$ & .589 \\
\hline (IC 95\%) & $(2.2-2.6)$ & $(2.0-2.5)$ & \\
\hline Lanzamiento de precisión & $2.1 \pm 0.7$ & $1.7 \pm 0.7$ & .001 \\
\hline (IC 95\%) & $(1.9-2.3)$ & $(1.4-1.8)$ & \\
\hline Golpeo de precisión & $2.0 \pm 0.6$ & $1.7 \pm 0.7$ & .019 \\
\hline (IC 95\%) & $(1.8-2.1)$ & $(1.4-1.8)$ & \\
\hline Eslalon & $2.6 \pm 1.0$ & $2.5 \pm 1.1$ & 616 \\
\hline (IC 95\%) & $(2.3-2.8)$ & $(2.1-2.7)$ & \\
\hline Bote & $2.1 \pm 1.1$ & $1.5 \pm 0.8$ & $<.001$ \\
\hline (IC 95\%) & $(1.9-2.3)$ & $(1.2-1.7)$ & \\
\hline Conducción & $1.9 \pm 0.9$ & $1.3 \pm 0.7$ & $<.001$ \\
\hline (IC 95\%) & $(1.7-2.1)$ & $(1.1-1.5)$ & \\
\hline \multicolumn{4}{|l|}{ Factores de coordinación motriz } \\
\hline Coordinación locomotriz & $8.2 \pm 1.8$ & $8.1 \pm 1.9$ & .905 \\
\hline (IC 95\%) & $(7.7-8.7)$ & $(7.6-8.7)$ & \\
\hline Coordinación control de objetos & $8.1 \pm 2.2$ & $6.1 \pm 2.2$ & $<.001$ \\
\hline (IC 95\%) & $(7.5-8.7)$ & $(5.4-6.7)$ & \\
\hline Coordinación control de objetos con el pie & $3.9 \pm 1.0$ & $2.9 \pm 1.2$ & $<.001$ \\
\hline (IC 95\%) & $(3.5-4.1)$ & $(2.6-3.3)$ & \\
\hline Coordinación control de objetos con la mano & $4.2 \pm 1.4$ & $3.1 \pm 1.1$ & $<.001$ \\
\hline (IC 95\%) & $(3.9-4.6)$ & $82.7-3.4)$ & \\
\hline Coordinación motriz global & $16.3 \pm 3.2$ & $14.2 \pm 3.3$ & .002 \\
\hline (IC 95\%) & $(15.4-17.2)$ & $(13.3-15.2)$ & \\
\hline Eficacia coordinativa & $.26 \pm .09$ & $.19 \pm .08$ & $<.001$ \\
\hline (IC 95\%) & $(.24-.28)$ & $(.17-.22)$ & \\
\hline Tiempo de ejecución (segundos) & $65.7 \pm 13.3$ & $77.9 \pm 17.7$ & $<.001$ \\
\hline (IC 95\%) & $(61.6-69.8)$ & $(73.3-82.3)$ & \\
\hline Actividad fisica & $2.9 \pm 0.8$ & $2.9 \pm 0.9$ & 998 \\
\hline (IC 95\%) & $(2.6-3.1)$ & $(2.6-3.1)$ & \\
\hline Menos activo, $n(\%)$ & $32(58.2)$ & $29(63.0)$ & .701 \\
\hline Más activo, $n(\%)$ & $23(41.8)$ & $17(37.0)$ & .343 \\
\hline
\end{tabular}

Fuente: elaboración propia.

Tabla 3

Diferencias en los parámetros de estudio según edad.

\begin{tabular}{lcccc}
\hline & $\begin{array}{c}6 \text { años } \\
(\mathrm{n}=31)\end{array}$ & $\begin{array}{c}7 \text { años } \\
(\mathrm{n}=46)\end{array}$ & $\begin{array}{c}8 \text { años } \\
(\mathrm{n}=24)\end{array}$ \\
\cline { 2 - 5 } & $\mathrm{M} \pm \mathrm{DT}$ & $\mathrm{M} \pm \mathrm{DT}$ & $\mathrm{M} \pm \mathrm{DT}$ & $p$ valor \\
\hline Pruebas del test motor & & & & \\
Salto vertical & $3.3 \pm 1.0$ & $3.2 \pm 1.1$ & $3.5 \pm 0.9$ & .388 \\
(IC 95\%) & $(2.9-3.6)$ & $(2.8-3.4)$ & $(3.1-3.9)$ & \\
Giro \%ongitudinal & $2.0 \pm 0.7$ & $2.3 \pm 0.8$ & $3.0 \pm 0.8$ & $<.001$ \\
(IC 95\%) & $(1.7-2.2)$ & $(2.1-2.5)$ & $(2.7-3.3)$ & \\
Lanzamiento de precisión & $1.8 \pm 0.7$ & $1.9 \pm 0.7$ & $2.0 \pm 0.8$ & .615 \\
(IC 95\%) & $(1.5-2.0)$ & $(1.7-2.1)$ & $(1.7-2.3)$ & \\
Golpeo de precisión & $1.7 \pm 0.7$ & $1.8 \pm 0.7$ & $2.0 \pm 0.7$ & .320 \\
(IC 95\%) & $(1.5-1.9)$ & $(1.5-1.9)$ & $(1.7-2.2)$ & \\
Slalom & $2.6 \pm 0.9$ & $2.5 \pm 1.1$ & $2.6 \pm 1.1$ & .782 \\
(IC 95\%) & $(2.2-2.9)$ & $(2.1-2.7)$ & $(2.1-3.0)$ & \\
Bote & $1.3 \pm 0.6$ & $1.9 \pm 0.9$ & $2.3 \pm 1.2$ & $<.001$ \\
(IC 95\%) & $(1.0-1.5)$ & $(1.6-2.2)$ & $(2.0-2.7)$ & \\
Conducción & $1.2 \pm 0.5$ & $1.6 \pm 0.6$ & $2.3 \pm 1.2$ & $<.001$ \\
(IC 95\%) & $(1.0-1.4)$ & $(1.4-1.8)$ & $(1.9-2.5)$ & \\
Factores de coordinación motriz & & & & \\
Coordinación locomotriz & $7.9 \pm 1.6$ & $7.9 \pm 1.8$ & $9.1 \pm 1.8$ & .025 \\
(IC 95\%) & $(7.2-8.5)$ & $(7.4-8.4)$ & $(8.3-9.8)$ & \\
Coord. control de objetos & $6.0 \pm 1.9$ & $7.3 \pm 1.9$ & $8.6 \pm 3.0$ & $<.001$ \\
(IC 95\%) & $(5.2-6.8)$ & $(6.6-7.9)$ & $(7.6-9.5)$ & \\
Coordinación control de objetos con el pie & $3.0 \pm 1.0$ & $3.4 \pm 1.0$ & $4.2 \pm 1.5$ & $<.001$ \\
(IC 95\%) & $(2.5-3.3)$ & $(3.1-3.7)$ & $(3.8-4.7)$ & \\
Coordinación control de objetos con la mano & $3.0 \pm 1.1$ & $3.8 \pm 1.2$ & $4.3 \pm 1.6$ & .002 \\
(IC 95\%) & $(2.6-3.5)$ & $(3.5-4.2)$ & $(3.8-4.8)$ & \\
Coordinación motriz global & $13.9 \pm 2.5$ & $15.2 \pm 3.0$ & $17.6 \pm 4.0$ & $<.001$ \\
(IC 95\%) & $(12.8-15.0)$ & $(14.3-16.1)$ & $(16.4-18.9)$ & \\
Eficacia coordinativa & $.17 \pm .05$ & $.24 \pm .07$ & $.29 \pm .11$ & $<.001$ \\
(IC 95\%) & $(.14-.20)$ & $(.22-26)$ & $(.26-32)$ & \\
Tiempo de ejecución (segundos) & $84.7 \pm 17.3$ & $65.2 \pm 9.7$ & $65.4 \pm 16.2$ & $<.001$ \\
(IC 95\%) & $(79.7-89.7)$ & $(61.1-69.3)$ & $(59.7-71.1)$ & \\
Actividad física & $2.7 \pm 1.0$ & $3.0 \pm 0.8$ & $2.8 \pm 1.0$ & .334 \\
(IC 95\%) & $(2.4-3.0)$ & $(2.7-3.2)$ & $(2.5-3.2)$ & \\
Menos activo, $n$ (\%) & $22(71.0)$ & $25(54.3)$ & $14(58.3)$ & .204 \\
Más activo, $n$ (\%) & $9(29.0)$ & $21(45.7)$ & $10(41.7)$ & .036 \\
\hline Fuente: elaboración propia. & & & & \\
& & & & \\
& & & & \\
& & &
\end{tabular}

ción $(p<.001)$, eficacia coordinativa $(p<.001)$ y sedentarismo $(p=.044)$ (tabla 3$)$. La prueba Chi-cuadrado arrojó diferencias estadísticamente en el nivel de actividad física, observándose una mayor prevalencia en el grupo más activo en la edad de 7 años frente a sus pares homólogos de 6 y 8 años $(p=.036)$.

El análisis de la relación entre actividad física y coordina-
Tabla

Relación entre el nivel de actividad fisica y la coordinación motriz

\begin{tabular}{|c|c|c|c|c|c|c|c|c|}
\hline & \multirow{2}{*}{\multicolumn{2}{|c|}{$\begin{array}{c}\text { Menos activo } \\
(\mathrm{n}=61)\end{array}$}} & \multicolumn{3}{|l|}{$\begin{array}{c}\text { Más activo } \\
(\mathrm{n}=40)\end{array}$} \\
\hline & & & & & & & & p valor \\
\hline \multicolumn{9}{|c|}{$\overline{P r u e b a s ~ d e l ~ t e s t ~ m o t o r ~}$} \\
\hline \multicolumn{4}{|l|}{$\begin{array}{l}\text { Salto vertical } \\
\text { (IC } 95 \%)\end{array}$} & $\begin{array}{l}3.2 \pm 1.1 \\
(2.9-3.5\end{array}$ & & $\begin{array}{r}3.3 \pm 0.9 \\
(3.0-3.7)\end{array}$ & & .483 \\
\hline \multicolumn{4}{|l|}{$\begin{array}{l}\text { Giro longitudinal } \\
\text { (IC } 95 \% \text { ) }\end{array}$} & $\begin{array}{l}2.3 \pm 0.9 \\
(2.1-2.5)\end{array}$ & & $\begin{array}{l}2.5 \pm 0.8 \\
(2.2-2.7)\end{array}$ & & .345 \\
\hline \multicolumn{4}{|c|}{$\begin{array}{l}\text { Lanzamiento de precisión } \\
\text { (IC } 95 \% \text { ) }\end{array}$} & $\begin{array}{l}1.8 \pm 0.7 \\
(1.6-2.0)\end{array}$ & & $\begin{array}{l}2.1 \pm 0.8 \\
(1.8-2.3)\end{array}$ & & .075 \\
\hline \multicolumn{4}{|c|}{$\begin{array}{l}\text { Golpeo de precisión } \\
\text { (IC } 95 \%)\end{array}$} & $\begin{array}{l}1.7 \pm 0.6 \\
(1.6-1.9)\end{array}$ & & $\begin{array}{l}1.9 \pm 0.7 \\
(1.7-2.1)\end{array}$ & & .344 \\
\hline \multicolumn{4}{|l|}{$\begin{array}{l}\text { Slalom } \\
\text { (IC 95\%) }\end{array}$} & $\begin{array}{l}2.5 \pm 0.9 \\
(2.2-2.7)\end{array}$ & & $\begin{array}{l}2.6 \pm 1.1 \\
(2.3-2.9)\end{array}$ & & .606 \\
\hline \multicolumn{4}{|l|}{$\begin{array}{l}\text { Bote } \\
\text { (IC 95\%) }\end{array}$} & $\begin{array}{l}1.5 \pm 0.8 \\
(1.3-1.8)\end{array}$ & & $\begin{array}{l}2.2 \pm 1.1 \\
(1.9-2.5)\end{array}$ & & . 001 \\
\hline \multicolumn{4}{|l|}{ Conducción } & $\begin{array}{l}1.4 \pm 0.7 \\
(1.2-1.6)\end{array}$ & & $\begin{array}{l}1.9 \pm 0.9 \\
(1.7-2.2)\end{array}$ & & .001 \\
\hline \multicolumn{9}{|c|}{$\begin{array}{l}\text { (IC 95\%) } \\
\text { Factores de coordinación motriz }\end{array}$} \\
\hline \multicolumn{4}{|c|}{$\begin{array}{l}\text { Coordinación locomotriz } \\
\text { (IC } 95 \% \text { ) }\end{array}$} & $\begin{array}{l}8.0 \pm 1.8 \\
(7.5-8.5)\end{array}$ & & $\begin{array}{l}8.4 \pm 1.8 \\
(7.8-9.0)\end{array}$ & & .268 \\
\hline \multicolumn{4}{|c|}{$\begin{array}{l}\text { Coordinación control de objetos } \\
\text { (IC } 95 \% \text { ) }\end{array}$} & $\begin{array}{l}6.5 \pm 2.0 \\
(5.9-7.1)\end{array}$ & & $\begin{array}{l}8.1 \pm 2.7 \\
(7.4-8.8)\end{array}$ & & .001 \\
\hline \multicolumn{4}{|c|}{$\begin{array}{l}\text { Coordinación control de objetos con el pie } \\
\text { (IC } 95 \% \text { ) }\end{array}$} & $\begin{array}{l}3.2 \pm 1.1 \\
(2.9-3.5)\end{array}$ & & $\begin{array}{l}3.8 \pm 1.3 \\
(3.5-4.2)\end{array}$ & & .005 \\
\hline \multicolumn{4}{|c|}{$\begin{array}{l}\text { Coordinación control de objetos con la mano } \\
\text { (IC } 95 \% \text { ) }\end{array}$} & $\begin{array}{l}3.3 \pm 1.1 \\
(3.0-3.7)\end{array}$ & & $\begin{array}{l}4.2 \pm 1.5 \\
(3.8-4.7)\end{array}$ & & .001 \\
\hline \multicolumn{4}{|c|}{$\begin{array}{l}\text { Coordinación motriz global } \\
\text { (IC } 95 \% \text { ) }\end{array}$} & $\begin{array}{r}14.6 \pm 3 . \\
(13.7-15 .\end{array}$ & & $\begin{array}{r}16.6 \pm 3.5 \\
(15.5-17.6)\end{array}$ & & .004 \\
\hline \multicolumn{4}{|c|}{$\begin{array}{l}\text { Eficacia coordinativa } \\
\text { (IC 95\%) }\end{array}$} & $(.21 \pm .08$ & & $(.27 \pm .09)$ & & .001 \\
\hline \multicolumn{4}{|c|}{$\begin{array}{l}\text { Tiempo de ejecución (segundos) } \\
\text { (IC 95\%) }\end{array}$} & $\begin{array}{l}75.0 \pm 17 \\
(71.0-79.6\end{array}$ & & $\begin{array}{l}65.5 \pm 13.4 \\
(60.5-70.5)\end{array}$ & & .004 \\
\hline \multicolumn{9}{|c|}{ Fuente: elaboración propia. } \\
\hline \multicolumn{9}{|l|}{ Tabla 5.} \\
\hline & \multicolumn{4}{|c|}{ Coordinación motriz global a } & \multicolumn{4}{|c|}{ Eficacia coordinativa $^{\mathrm{b}}$} \\
\hline & B & $\mathrm{EE}$ & $r$ & $p$ & B & $\mathrm{EE}$ & $r$ & $P$ \\
\hline Género & -1.830 & .594 & -299 & .003 & -.058 & .014 & -.394 & $<.<$. \\
\hline Edad (años) & 1.675 & .403 & .389 & $<.001$ & .054 & .009 & .504 & $<.001$ \\
\hline Actividad física & .765 & .340 & .223 & .027 & .030 & 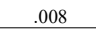 & .360 & $<.0$ \\
\hline
\end{tabular}

ción motriz se puede observar en la tabla 4. La prueba ANOVA detectó diferencias significativas en las variables bote ( $p=$ $0,001)$, conducción ( $p=.001), C C O(p=0.001), C C O P(p=$ $.005), C C O M(p=.001), C M G(p=.004)$, tiempo de ejecución $(p=.004)$ y eficacia coordinativa $(p=.001)$.

Como análisis complementario y con el propósito de comprobar la capacidad predictiva de la actividad física se realizó un análisis de regresión lineal (tabla 5). La actividad física se asoció de manera positiva con la CMG ( $\beta$ no estandarizada $=$ $0.765, p=.027$ ) tras ajustar por género y edad. La edad también se relacionó de forma positiva con la CMG, observándose que a mayor edad mejoraba la $\mathrm{CMG}(\beta$ no estandarizada $=1.675, p<.001)$, mientras que el sexo se asoció de manera inversa con la CMG, encontrándose que las mujeres presentaron una menor $\mathrm{CMG}(\beta$ no estandarizada $=-1.830, p=.003)$. La actividad física también se relacionó positivamente con la eficacia coordinativa ( $\hat{a}$ no estandarizada $=-.030, r=.360, p<$ $.001)$ tras ajustar por género y edad. Se observó que el género se asoció con la eficacia coordinativa (â no estandarizada $=-.058, r=-.394, p<.001)$ mostrando los varones registros superiores de eficacia coordinativa. Por último, la edad también se relacionó con la eficacia coordinativa (â no estandarizada $=.054, r=.504, p<.001$ ).

\section{Discusión}

Este estudio analizó en 101 escolares españoles de seis a ocho años de edad la coordinación motriz según género, edad y nivel de actividad física, aplicando un instrumento que permite examinar la coordinación tanto en su globalidad como en algunas de sus expresiones como la coordinación locomotriz y la coordinación control de objetos con las manos y con los pies.

Con respecto al análisis de la coordinación motriz según 
género, los resultados indican que los varones tienen un mejor rendimiento coordinativo en las tareas de lanzamiento de precisión, golpeo de precisión, bote y conducción, mostrando en consecuencia registros superiores en los factores de CCO, CCOP, CCOM, CMG y eficacia coordinativa.

Estos resultados son coherentes, en general, con los hallazgos encontrados en diversas investigaciones (ComukBalci, et al., 2016; Torralba, et al., 2016; Yanci \& los Arcos, 2015; Yanci, et al., 2014; Tekok-Kiliç, et al., 2010; Lopes, et al., 2003) y, en concreto, en habilidades relacionadas con la puntería y control de objetos (Bravo, et al., 2017; FernándezLosa, et al., 2013), en la velocidad (De Miguel-Etayo, et al., 2014), o en lanzamiento de peso (Carrillo-López, et al., 2018). Además, coincide en cierta medida con lo reportado por Jones \& Lorenzo (2013) y Carrillo-López, et al. (2018), los cuales observaron una asociación consistente entre dos cualidades físicas relacionadas con la coordinación locomotriz, como son la velocidad y la agilidad.

Por el contrario, algunos estudios o bien no han encontrado diferencias por género (Coetezee \& Du Plessis, 2013; Singh, et al., 2010), o bien han reportado diferencias solo en algunas de las pruebas como la velocidad, la agilidad o el lanzamiento de peso, no registrando diferencias en pruebas como el desplazamiento sobre soportes o el salto sobre un apoyo (Carrillo-López et al., 2018). En este sentido, un estudio observó un mejor rendimiento coordinativo en las mujeres en habilidades como el salto, tanto a una pierna como a dos piernas Coetezee (2016); aunque respecto a esta última habilidad este trabajo es coherente con otros estudios puesto que los varones manifestaron mayor capacidad de salto que las mujeres (Jones \& Lorenzo, 2013; López, et al., 2016; Piernar, et al., 2012). Estas diferencias podrían ser debidas a las preferencias de actividad física y deporte, teniendo los varones un mejor rendimiento en aquellas pruebas donde se incluye un componente de equilibrio dinámico (RodríguezNegro \& Yanci, 2019).

En relación al análisis de la coordinación motriz según la edad, los resultados de este estudio confirman la existencia de diferencias significativas. Existe una tendencia generalizada de estudios donde se asocia un mayor nivel de coordinación motriz en parámetros como el salto conforme se incrementa la edad (Torres-Luque, et al., 2014; López, et al., 2016). A pesar de ello, Yanci, et al. (2014) cuestionan la utilidad de establecer diferencias a estas edades, debido a que en muchas de estas habilidades se produce una mejora generalizada de los patrones motores generales en ambos sexos. Según Marta, et al. (2012) esto podría estar determinado por factores genéticos asociados al crecimiento.

Los resultados de este estudio indican que los escolares con un mayor nivel de actividad física presentan un mejor rendimiento físico en las tareas de bote y conducción, así como valores superiores en los factores de CCO, CCOP, CCOM, CMG y eficacia coordinativa.

Estos resultados coinciden en gran medida con lo reportado por Ericsson (2011) en un estudio longitudinal de nueve años de duración realizado con 263 escolares suecos de siete años. Tras solo un año de intervención, el grupo que recibía cinco sesiones semanales de educación física escolar ya mostraba un mejor desempeño coordinativo que aquellos que solo realizaban dos sesiones semanales. Estas diferen- cias se mantuvieron a los 15 años confirmándose la hipótesis de que el entrenamiento favorece un mejor desarrollo motor del individuo, sobre todo, en equilibrio y coordinación motriz.

Relacionado con estos hallazgos, una investigación realizada con escolares de seis a nueve años concluyó que una intervención metodológica en el área de educación física con un mayor nivel de compromiso motor favorecía el desarrollo cualitativo de las habilidades motrices básicas y, por ende, en la coordinación motriz (Jarani, et al., 2016). En la misma línea, otro estudio longitudinal de nueve años de duración realizado, en este caso, con 389 escolares albanos de seis a nueve años, determinó que los escolares que recibían más horas de educación física o habían gozado de una enseñanza adaptada a sus capacidades presentaban mejores habilidades motrices, desarrollo coordinativo, e incluso obtenían resultados académicos superiores (Ericsson \& Karlsson, 2014).

En el estudio de Walhain, et al. (2016) en escolares de siete años de Surinam procedentes de un entorno rural informaron de niveles superiores de actividad física en comparación con sus semejantes de entorno urbano, mostrando a su vez registros superiores de capacidad aeróbica, competencia motriz gruesa (caminar hacia atrás y saltando hacia los lados), y competencia motriz fina (destrezas manuales), aunque tuvieron registros inferiores de competencia motriz gruesa (desplazamiento lateral).

Los resultados de estos estudios ponen de manifiesto que, cuando los escolares presentan niveles similares de actividad física, el entorno sociocultural en el cual se desarrollan no parece ser un factor tan determinante en el desarrollo de la coordinación motriz, por lo que se deberían analizar de manera conjunta otros factores. Por ejemplo, uno de estos factores podría ser el nivel de condición física que poseen. En este sentido, aunque se ha observado una relación directa y bidireccional entre la actividad física y la condición física (Rosa, et al., 2017) y, que esta última, se relaciona con un mejor desempeño motor en pruebas que miden la coordinación dinámica general, la velocidad-agilidad y la capacidad de salto horizontal (López, et al., 2016; TorresLuque, et al., 2014; Rosa, et al., 2015; Rosa \& García-Cantó, 2017; Ru•barská, 2016; Secchi, et al., 2014), un reciente trabajo ha revelado resultados poco consistentes en la relación entre condición física, nivel de actividad física y rendimiento coordinativo (Hardman, et al., 2017). La realización de estudios prospectivos y de corte longitudinal podría aportan información más consistente sobre el modo en el que el tipo y la frecuencia de actividad física puede influir sobre el desarrollo de la coordinación motriz de los individuos a lo largo de su escolarización y en periodos vitales posteriores.

Este estudio no se encuentra exento de limitaciones. El carácter descriptivo y transversal dificulta, por un lado, el establecimiento de relaciones de tipo causal entre las variables y, por otro lado, el análisis de la evolución de esta relación a largo plazo. También es necesario señalar que el análisis de la coordinación motriz se ha realizado mediante pruebas extraídas de un test motor que no aporta la misma precisión que las medidas realizadas en laboratorio. No obstante, el Test 3JS es un instrumento que aporta una buena validez, confiabilidad y aplicabilidad para evaluar la coordinación 
motriz en poblaciones pediátricas. En este sentido, una de las fortalezas de este trabajo es el aporte de hallazgos relevantes sobre las distintas dimensiones de la coordinación motriz, incluyendo la eficacia coordinativa, en edades relevantes para el desarrollo motor.

En conclusión, los resultados de este estudio indican que los escolares con un mayor nivel de actividad física tienen una mejor coordinación motriz, lo que sugiere que estructurar programas de actividad física orientados a este objetivo podría reportar resultados de gran interés a nivel motor, físico y educativo. Los varones mostraron una mayor coordinación motriz, lo que indica la necesidad de fomentar programas que faciliten la adherencia a la actividad física para mujeres en edad escolar por las implicaciones anteriormente señaladas. La edad y la coordinación motriz también se encuentran asociados, encontrándose que los escolares de ocho años tienen una mejor coordinación motriz que sus semejantes de seis y siete años, lo que confirma la necesidad de impulsar programas de actividad física adaptados al desarrollo madurativo.

\section{Referencias}

Barnett, L. M., Van Beurden, E., Morgan, P. J., Brooks, L.O., \& Beard, J.R. (2010). Gender differences in motor skill proficiency from childhood to adolescence:Alongitudinal study. Research Quarterly for Exercise and Sport, 81,162-170.

Bravo, I., Rodríguez-Negro, J., \& Irigoyen, J. Y. (2017). Diferencias en función del género en la puntería y atrape en niños de Educación Primaria. Retos, (32), 35-38.

Bucco, L., \& Zubiaur, M. (2015). Analysis of the Motor Development in Brazilian Schoolchildren with Corporal Measures of Obesity and Overweight. Revista Internacional de Medicina y Ciencias de la Actividad Física y el Deporte, 15(59), 593-611.

Butterfield, S. A., Angell, R. M., \& Mason, C.A. (2012).Age and sex differences in object control skills by children ages 5 to 14. Perceptual and Motor Skills, 114, 261-274.

Cairney, J. (Ed.) (2015). Developmental Coordination Disorders and its consequences. Toronto: University of Toronto Press.

Cairney, J., Hay, J.A., Faught, B.E., Mandigo, J., \& Flouris, A. (2005a). Developmental Coordination Disorder, Self-Efficacy Toward Physical Activity and Participation in Free Play and Organized Activities: Does Gender Matter? Adapted Physical Activity Quarterly, 22(1), 67-82.

Cairney, J., Hay, J.A., Faught, B.E., Wade, T.J., Corna, L., \& Flouris, A. (2005b). 'Developmental Coordination Disorder, Generalized Self-Efficacy Toward Physical Activity and Participation in Organized and Free Play Activities'. Journal of Pediatrics, 147(4), 515-20.

Carrillo-López,P.J., Rosa-Guillamón,A., \& García-Cantó,E. (2018). Análisis de la coordinación motriz global en escolares de 6 a 9 años atendiendo al género y edad. Trances, 10(3) ,281-306.

Cenizo, J. M., Ravelo, J., Morilla, S., \& Fernández-Truan, J. C. (2017). Test de coordinación motriz 3JS: Cómo valorary analizar su ejecución. Retos, (32), 189-193.

Cenizo, J. M., Ravelo, J., Morilla, S., Ramírez, J. M., \& FernándezTruan, J. C. (2016). Diseño y validación de instrumento para evaluar coordinación motriz en Primaria. Revista Internacional de Medicina y Ciencias de la Actividad Física y del Deporte, 16(62), 203-219.

Cenizo, J. M., Ravelo, J., Ramírez, J. M., \& Fernández, J. C. (2015).
Assessment of Motor Coordination in Students Aged 6 to 11 Years. Journal of Physical Education and Sport, 15(14), 765-774.

Coetzee, D. (2016). Strength, running speed, agility and balance profiles of 9-to 10-year-old learners: NW-child study. South African Journal for Research in Sport, Physical Education and Recreation, 38(1), 13-30.

Coetzee, D., \& Du Plessis, W. (2013). Visual-motor status of Grade 1 learners in the North-West province of South Africa: NWChild study. South African Journal for Research in Sport, Physical Education and Recreation, 35(2), 37-50.

Comuk-Balci, N., Bayoglu, B., Tekindal,A., Kerem-Gunel, M., \& Anlar, B. (2016). Screening preschool children for fine motor skills: environmental influence. Journal of Physical Therapy Science, 28(3), 1026-1031.

De Meester,A., Stodden, D., Brian, A., True, L., Cardon, G., Tallir, I., et al. (2016) Associations among Elementary School Children's Actual Motor Competence, Perceived Motor Competence, Physical Activity and BMI: A Cross-Sectional Study. PLOS ONE, 11(10).

De Miguel-Etayo, P., Gracia-Marco, L., Ortega, F. B., Intemann, T., Foraita, R., Lissner, L., .. \& \& Moreno, L.A. (2014). Physical fitness reference standards in European children: the IDEFICS study. International Journal of Obesity, 38, S57-S66.

Ericsson, I. (2011). Effects of increased physical activity on motor skills and marks in physical education: an intervention study in school years 1 through 9 in Sweden. Physical Education \& Sport Pedagogy, 16(3), 313-329.

Ericsson, I., \& Karlsson, M. K. (2014). Motor skills and school performance in children with daily physical education in school-a 9 year intervention study. Scandinavian journal of medicine \& science in sports, 24(2), 273-278.

Fernández-Losa, J., Cecchini, J.A., \& Pallasá, M. (2013). La recepción de balón en niños con edades comprendidas entre los 3 y los 12 años. Revista Internacional de Medicina y Ciencias de la Actividad Física y del Deporte, 13, 279-294.

Freitas, D., Lausen, B., Maia, J., Lefevre, J., Rubio, E., Thomis, M., ... \& Malina, R.M. (2015). Skeletal maturation, fundamental motor skills and motor coordination. Journal of Sports Sciences, 33(9), 924-934.

Gallahue, D.L., \& Ozmun, J.C. (2006). Understanding motor development: Infants, children, adolescents, adults (6th ed.). Dubuque, IA: McGraw-Hill.

Gorla, J. I.,Araújo, P. F. d., \& Rodrigues, J. L. (2010). Evaluación Motoras en Educación Física Adaptada: Test KTK (2. ${ }^{a}$ ed.). São Paulo: Phorte.

Hardman, C. M., Júnior, W., de Souza, R., Oliveira, E. S. A. D., \& Barros, M. V. G. D. (2017). Relationship between physical activity and BMI with level of motor coordination performance in schoolchildren. Revista Brasileira de Cineantropometria \& Desempenho Humano, 19(1), 50-61.

Hay, J. (1996). Predicting the Selection of Physical Education Class in Grade 10 from Self-Perceptions Reported in Grades Seven, Eight, and Nine. BrockEducation6: 59-69.

Henderson, S. E., Sugden, D., \& Barnett, L. (2007). Movement Assessment Battery for Children-second edition. Londres: Pearson Assessment.

Jarani, J., Grøntved,A., Muca, F., Spahi,A., Qefalia, D., Ushtelenca, K., ... \& Gallotta, M. C. (2016). Effects of two physical education programmes on health-and skill-related physical fitness of Albanian children. Journal of sports sciences, 34(1), 
$35-46$.

Jones, M.T., \& Lorenzo, D.C.(2013). Assessment of power, speed, and agility in athletic, preadolescent youth. The Journal of Sports Medicine and Physical Fitness, 53(6), 693 - 700.

Kiphard, E. J., \& Schilling, V. F. (1974). Körper-koordinationstest für kinder KTK: manual Von Fridhelm Schilling. Weinhein: Beltz Test.

Kokštejn, J., Psotta, R., \& Musálek, M. (2015). Motor competence in Czech children aged 11-15: What is the incidence of a risk of developmental coordination disorder? Acta Gymnica, 45(2), 61-68.

Lazzer, S., Pozzo, R., Rejc, E., Antonutto, G., \& Francescato, M.P. (2009). Maximal explosive muscle power in obese and nonobese prepubertal children. Clinical Physiology Functional Imaging, 29(3), 224-228.

Lingam, R., Hunt, L., Golding, J., Jongmans, M., \& Emond, A (2009). Prevalence of Developmental Coordination Disorder Using the DSM-IV at 7 Years of Age: A UK PopulationBased Study. Pediatrics, 123(4), 693-700.

Lladó, J. (2017). Relación Entre el Body Mass Index y la Coordinación Motriz en Alumnos y Alumnas de Educación Primaria. Revista de educación física: Renovar la teoría y práctica, 35(2), 12-20.

López, F. J., Lara,A. J., Espejo, N., \& Cachón, J. (2016). Influencia del género, la edad y el nivel de actividad física en la condición física de alumnos de educación primaria. Revisión Bibliográfica. Retos, (29), 129-133.

Lopes, V.P., Maia, J.A. R., Silva, R. G., Seabra,A., \& Morais, F. P. (2003). Estudo do nível de desenvolvimento da coordenação motora da população escolar (6 a 10 años de idade) da Região Autónoma dos Açores. Revista Portuguesa de Ciências do Desporto, 3(1), 47-60.

Marta, C.C., Marinho, D.A., Barbosa, T.M., Izquierdo, M., \& Marques, M.C. (2012). Physical fitness differences between prepubescent boys and girls. Journal of Strength and Conditioning Research, 26(7), 1756-1766.

Meinel, K., \& Schnabel, G. (2013). Teoría del movimiento: Motricidad deportiva 8 (2. ${ }^{a}$ ed). Buenos Aires: Editorial StadiumSRL.

Pérez-Soto, J.J, García-Cantó, E., Rosa-Guillamón, A., RodríguezGarcía,P.L.,Moral-García,J.E., \& López-García,S.(2018).Afterschool leisure time: physical activity and estimated caloric expenditure in schoolchildren from southeast Spain. Rev. Fac. Med., 66(2), 209-14.

Picq, L. \& Vayer, P. (1977). Educación Psicomotriz. Barcelona: Científico.

Pienaar,A.E., Du Toit, D., Stickling,A., Peens,A., Botha, J., Kemp, C., et al. (2012). Motor development, growth, motor deficiencies, the assessment and intervention thereof: $\mathrm{Ma}$ nual for postgraduate students in Kinderkinetics. Potchefstroom, RSA: Xerox Noordwes Universiteit.

Rodríguez-Negro, J., \& Yanci, J. (2019). Diferencias en función del género en el equilibrio estático y dinámico en estudiantes de educación primaria. Retos, 35, 113-116.

Román, B., Serra, L., Ribas, L., Pérez, C., \& Aranceta J. (2003). Estimación del nivel de actividad física mediante el Test Corto Krece Plus. Resultados de la población española. En: Crecimiento y desarrollo. Estudio en Kid. Ed. Serra, L. \& Aranceta. J. (pp.59-74). Barcelona: Masson.

Rosa,A., \& García-Cantó, E. (2017). Relationship between muscle strength and other parameters of fitness in primary school children, SportTK: Revista Euroamericana de Ciencias del Deporte, 6(1), 107-116.

Rosa, A., García-Cantó, E., \& Carrillo, P. J. (2018). Percepción de salud, actividad física y condición física en escolares. Cuadernos de Psicología del Deporte, 18(3), 179-189.

Rosa,A., García-Cantó, E., \& Carrillo-López, P.J. (2019). Capacidad aeróbica y rendimiento académico en escolares de educación primaria. Retos, 35, 351-354.

Rosa,A., García, E., \& Pérez, J.J. (2018). Condición física y bienestar emocional en escolares de 7 a 12 años. Acta. colomb. psicol., 21(2), 282-291.

Rosa,A., García-Cantó, E., Rodríguez-García, P.L., \& Pérez-Soto, J.J. (2015). Aerobic capacity and its relationship with parameters of health-related fitness in schoolchildren. Rev. Fac. Cienc. Salud UDES, 2(2),90-6.

Rosa, A., García-Cantó, E., Rodríguez-García, P.L., Pérez-Soto, J.J., Tárraga-Marcos, M.L., \& Tárraga-López, P.J.(2017). Actividad física, condición física y calidad de la dieta en escolares de 8 a 12 años. Nutr. Hosp., 34(6), 1292-8.

Ruiz-Pérez, L. M., Navia, J., Ruiz, A., Ramón, I., \& Palomo, M. (2016). Coordinación motriz y rendimiento académico en adolescentes. Retos, (29), 86-89.

Ruiz-Pérez,L. M., Rioja-Collado, N., Graupera-Sanz, J.L., PalomoNieto, M. \& Garcia-Coll, V.(2015). Grami-2: desarrollo de un test para evaluar la coordinación motriz global en la educación primaria. Revista Iberoamericana de Psicología del Ejercicio y el Deporte, 10(1), 103-111.

Ru•barská, I. (2016). Physical fitness of primary school children in the reflection of different levels of gross motor coordination. Acta Gymnica, 46(4),184-192.

Secchi, J. D., García, G. D., España-Romero, V., \& Castro Piñero, J. (2014). Condición física y riesgo cardiovascular futuro en niños y adolescentes argentinos: una introducción de la Batería ALPHA. Archivos Argentinos de Pediatría, 112(2), 132140.

Singh, C.K., Dhanda, B., \& Shanwal, P. (2010). Gender difference in motor and mental development in children: An impact of stimulating activities. Anthropologist, 12(2), 153-154.

Tekok-Kiliç,A., Elmastaç-Dikeç, E., \& Can, H. (2010). Evaluation of visual-motor integration functions in children between 615 years of age. Turkish Journal of Psychiatry, 21(2), 97-104.

Torralba, M.A., Vieira, M. B., Lleixà, T., \& Gorla, J. I. (2016). Evaluación de la coordinación motora en educación primaria de Barcelona y provincia. Revista Internacional de Medicina y Ciencias de la Actividad Física y del Deporte, 16(62), 355-371.

Torres-Luque, G, Carpio, E., Lara, A., \& Zagalaz, M. a L. (2014). Niveles de condición física de escolares de educación primaria en relación a su nivel de actividad física y al género. Retos, $25,17-22$.

Walhain, F., van Gorp, M., Lamur, K. S., Veeger, D. H., \& Ledebt, A. (2016). Health-Related Fitness, Motor Coordination, and Physical and Sedentary Activities of Urban and Rural Children in Suriname. Journal of physical activity and health, 13(10), 1035-1041.

Yanci, J., \& Los Arcos, A. (2015). How does the age and gender influence the ability to change direction in primary school children? Retos, 28, 40-43.

Yanci, J., Los Arcos, A., Reina, R., Gil, E., \& Grande, I. (2014). Agility in primary education students: Differences by age and gender. International Journal of Medicine and Science of Physical Activity and Sport, 14(53), 23-35. 